\title{
THE COMPLETE PRODUCT OF ANNIHILATINGLY UNIQUE DIGRAPHS
}

\author{
C. S. GAN
}

Received 25 February 2005 and in revised form 10 May 2005

Let $G$ be a digraph with $n$ vertices and let $A(G)$ be its adjacency matrix. A monic polynomial $f(x)$ of degree at most $n$ is called an annihilating polynomial of $G$ if $f(A(G))=0$. $G$ is said to be annihilatingly unique if it possesses a unique annihilating polynomial. Difans and diwheels are two classes of annihilatingly unique digraphs. In this paper, it is shown that the complete product of difan and diwheel is annihilatingly unique.

\section{Introduction}

All graphs under consideration in this paper are directed, connected, finite, loopless, and without multiple arcs. By a digraph $G=(V, E)$, we mean a finite set $V$ (the elements of which are called vertices) together with a set $E$ of ordered pairs of elements of $V$ (these pairs are called arcs). Two vertices are said to be adjacent if they are connected by an arc. Undefined terms and notations can be found in $[1,2,4]$.

A diwalk in a digraph is an alternating sequence of vertices and arcs, $v_{0}, x_{1}, v_{1}, \ldots, x_{k}, v_{k}$ in which each arc $x_{i}$ is $\left(v_{i-1}, v_{i}\right)$. The length of such diwalk is $k$, the number of occurrences of arcs in it.

A dipath $P_{n}$ is a diwalk of order $n$ in which no vertex is repeated.

A directed cycle $C_{n}$ of order $n$ is a digraph with vertex set $\left\{v_{1}, \ldots, v_{n}\right\}$ having arcs $\left(v_{i}, v_{i+1}\right), i=1,2, \ldots, n-1$, and $\left(v_{n}, v_{1}\right)$.

A difan $F_{n}$ is a digraph consisting essentially of a dipath $P_{n-1}$ of $n-1$ vertices labelled $1,2, \ldots, n-1$ and an additional vertex $n$, where there is an $\operatorname{arc}$ from $n$ to each of the vertices of $P_{n-1}$. Vertex $n$ is called the $h u b$ of the difan whereas the $\operatorname{arc}(n, k)$ from the hub $n$ to the vertex $k$ of $P_{n-1}$ is called a spoke. Vertices with labelling $i=1,2, \ldots, n-1$ are called the rim vertices of the difan.

The diwheel $W_{n}$ of order $n$ consists of a directed cycle $C_{n-1}$ with an additional vertex $n$ joined from it to all the others. Vertex $n$ is called the hub of the diwheel, whereas the $\operatorname{arc}(n, k)$ from the hub $n$ to the vertex $k$ of $C_{n-1}$ is called a spoke. Vertices with labelling $i$ where $i=1,2, \ldots, n-1$ are called the rim vertices of the diwheel.

Let $G$ be a digraph with $n$ vertices. The adjacency matrix $A(G)=\left(a_{i j}\right)$ of $G$ is a square matrix of order $n$ where the $(i, j)$-entry, $a_{i j}$, is equal to the number of arcs starting at 


\section{The complete product of annihilatingly unique digraphs}

the vertex $i$ and terminating at the vertex $j$. Let $A^{k}(G)=\left(a_{i j}^{k}\right)$ where $k$ is a positive integer and the $(i, j)$-entry $a_{i j}^{k}$ of $A^{k}(G)$ is the number of different diwalks at length $k$ from the vertex $i$ to vertex $j$.

The determinant of a square matrix $A$ is denoted by $|A|$ or (det) $A$. The characteristic polynomial $|x I-A(G)|$ of the adjacency matrix $A(G)$ of $G$ is called the characteristic polynomial of $G$ and is denoted by $\psi(x)$. A monic polynomial $f(x)$ of degree at most $n$ with $f(A(G))=0$ is called an annihilating polynomial of $G$. The existence of annihilating polynomial of $G$ is guaranteed by its characteristic polynomial. $G$ is said to be annihilatingly unique if it possesses a unique annihilating polynomial.

The following result is well known in linear algebra (see $[1,5,6])$.

Theorem 1.1. Let $A$ be an $n \times n$ matrix, $m(x)$ its minimum polynomial, and $\psi(x)$ its characteristic polynomial.

(1) $\psi(A)=0$.

(2) If $f(x)$ is any polynomial with $f(A)=0$, then $m(x)$ divides $f(x)$; in particular $m(x)$ divides $\psi(x)$.

(3) Let $\left\{x_{1}, x_{2}, \ldots, x_{k}\right\}$ be the set of distinct eigenvalues of $A, x_{i}$ having algebraic multiplicity $c_{i}$. Then

$$
\begin{aligned}
\psi(x) & =\left(x-x_{1}\right)^{c_{1}}\left(x-x_{2}\right)^{c_{2}} \cdots\left(x-x_{k}\right)^{c_{k}}, \\
m(x) & =\left(x-x_{1}\right)^{q_{1}}\left(x-x_{2}\right)^{q_{2}} \cdots\left(x-x_{k}\right)^{q_{k}},
\end{aligned}
$$

where $q_{i}$ satisfies $0<q_{i} \leq c_{i}(1,2, \ldots, k)$. Furthermore, if $k=n$, then

$$
m(x)=\psi(x)=\left(x-x_{1}\right)\left(x-x_{2}\right) \cdots\left(x-x_{n}\right) .
$$

A matrix for which the minimum polynomial is equal to the characteristic polynomial is called nonderogatory; otherwise derogatory. The following result follows from Theorem 1.1.

THeOREM 1.2. The annihilating polynomial $f(x)$ of any digraph $G$ with adjacency matrix $A(G)$ is unique if and only if $A(G)$ is nonderogatory.

Corollary 1.3. Let $G$ be a digraph with $n$ vertices and $A(G)$ its adjacency matrix. Then $G$ is annihilatingly unique if $A(G)$ has $n$ distinct eigenvalues.

\section{The complete product of digraphs}

In this section, we will consider a binary operation on digraphs: the complete product of digraphs.

The direct sum $G_{1}+G_{2}$ of digraphs $G_{1}\left(V_{1}, E_{1}\right)$ and $G_{2}\left(V_{2}, E_{2}\right)\left(V_{1} \cap V_{2}=\varnothing\right)$ is the digraph $G(V, E)$ for which $V=V_{1} \cup V_{2}$ and $E=E_{1} \cup E_{2}$.

The complete product $G_{1} \otimes G_{2}$ of digraphs $G_{1}$ and $G_{2}$ is the digraph obtained from $G_{1}+G_{2}$ by joining every vertex of $G_{1}$ to every vertex of $G_{2}$.

Let $G_{1}$ and $G_{2}$ be digraphs with $n_{1}$ and $n_{2}$ vertices, respectively. The vertices of $G_{1} \otimes G_{2}$ are labelled as follows. 
The vertices of $G_{2}$ are labelled $1,2, \ldots, n_{2}$ and vertices of $G_{1}$ are labelled $n_{2}+1, n_{2}+$ $2, \ldots, n_{2}+n_{1}$. Suppose that $A\left(G_{i}\right)$ is the adjacency matrix of $G_{i}, i=1,2$. Then the adjacency matrix of $G_{1} \otimes G_{2}$ is of the form

$$
\left[\begin{array}{cc}
A\left(G_{2}\right) & 0_{n_{1}}^{\left(n_{2}\right)} \\
J_{n_{2}}^{\left(n_{1}\right)} & A\left(G_{1}\right)
\end{array}\right],
$$

where $J_{n_{2}}^{\left(n_{1}\right)}$ is an $n_{1} \times n_{2}$ matrix with all entries equal to 1 and $0_{n_{1}}^{\left(n_{2}\right)}$ is an $n_{2} \times n_{1}$ matrix with all the entries equal to zero. It follows immediately that the characteristic polynomial of $G_{1} \otimes G_{2}$ is the product of the characteristic polynomials of $G_{1}$ and $G_{2}$.

A question arises: is it true that the complete product of two annihilatingly unique digraphs possesses a unique annihilating polynomial?

The complete product of annihilatingly unique digraphs was first studied by Lam [3]. In particular, Lam proved that $P_{q} \otimes P_{r}, P_{q} \otimes C_{r}, C_{r} \otimes P_{q}, P_{q} \otimes W_{r}, W_{r} \otimes P_{q}$ are annihilatingly unique. Furthermore, if $q$ and $r$ are relatively prime, then $C_{q} \otimes C_{r}, C_{q} \otimes W_{r+1}$, $W_{r+1} \otimes C_{q}$, and $W_{q+1} \otimes W_{r+1}$ are annihilatingly unique.

In general, it is not true that the complete product of two annihilatingly unique digraphs possesses a unique annihilating polynomial. For example, $C_{3} \otimes W_{4}$ of order 7 is not annihilatingly unique.

Motivated by the above results, we will prove that the complete product of difans and diwheels are annihilatingly unique in the next section.

\section{The complete product of difans and diwheels}

In this section, we show that the complete products $F_{q} \otimes W_{r}$ and $W_{q} \otimes F_{r}$ of order $q+r$ are annihilatingly unique. The characteristic polynomials for difan of order $q$ and diwheel of order $r$ are $x^{q}$ and $x^{r}-x$, respectively.

Theorem 3.1. $F_{q} \otimes W_{r}$ and $W_{q} \otimes F_{r}$ are annihilatingly unique.

Proof. We divide the proof of Theorem 3.1 into two parts and in each part, we will prove that the given complete product of digraphs is annihilatingly unique by showing the equality of its characteristic and minimum polynomials.

Part $1\left(F_{q} \otimes W_{r}\right.$ is annihilatingly unique). The characteristic polynomial of $F_{q} \otimes W_{r}$ is given by $\psi(x)=x^{q}\left(x^{r}-x\right)=x^{q+1}\left(x^{r-1}-1\right)$. From the digraph of $F_{q} \otimes W_{r}$, we observe that $a_{r, r-1}^{(r-1)}=1$ and $a_{r+q, 1}^{(q+1)}>1$ (this is because there exist at least two diwalks of length $q+1$ from vertex $r+q$ to vertex 1 . For example, $r+q \rightarrow r+1 \rightarrow r+2 \rightarrow r+3 \rightarrow \cdots \rightarrow$ $r+q-2 \rightarrow r+q-1 \rightarrow r \rightarrow 1$ and $r+q \rightarrow r+1 \rightarrow r+2 \rightarrow r+3 \rightarrow \cdots \rightarrow r+q-2 \rightarrow r+$ $q-1 \rightarrow r-1 \rightarrow 1$ are two such diwalks of length $q+1$ from vertex $r+q$ to vertex 1$)$. This observation implies that $A^{q+1}$ and $A^{r-1}-I$ are not equal to 0 and $x^{q+1}$ and $x^{r-1}-1$ are therefore not the minimum polynomials of $\psi(x)$.

Notice that the only repeated factor of the characteristic polynomial of $F_{q} \otimes W_{r}$ is $x$ of multiplicity $q+1$. We claim that $\psi(x)=m(x)=x^{q+1}\left(x^{r-1}-1\right)$. Suppose that $m(x)=$ $x^{q}\left(x^{r-1}-1\right)=x^{q+r-1}-x^{q}$. We will show that $m(A) \neq 0$ to obtain a contradiction. From the digraph of $F_{q} \otimes W_{r}$, we observe that $a_{r+q, r}^{(r+q-1)}=0$ and $a_{r+q, r}^{(q)}=1$. This implies that 
$m(A)=A^{q+r-1}-A^{q} \neq 0$, a contradiction. Therefore, $\psi(x)$ is equal to the minimum polynomial of $F_{q} \otimes W_{r}$. Hence, $F_{q} \otimes W_{r}$ is annihilatingly unique.

Part $2\left(W_{q} \otimes F_{r}\right.$ is annihilatingly unique). The characteristic polynomial of $W_{q} \otimes F_{r}$ is given by $\psi(x)=x^{r}\left(x^{q}-x\right)=x^{r+1}\left(x^{q-1}-1\right)$. Like the case of $F_{q} \otimes W_{r}$, from the digraph of $W_{q} \otimes F_{r}$, we have $a_{r+q, r+q-1}^{(q-1)}=1$ and $a_{r+1, r-1}^{(r+1)}>1$. This implies that $A^{r+1}$ and $A^{q-1}-I$ are not equal to 0 and $x^{r+1}$ and $x^{q-1}-1$ are therefore not the minimum polynomials of $\psi(x)$.

Notice that the only repeated factor of $\psi(x)$ is $x$ of multiplicity $r+1$. We claim that $\psi(x)=m(x)=x^{r+1}\left(x^{q-1}-1\right)$. Suppose on the contrary that $m(x)=x^{r}\left(x^{q-1}-1\right)$. Again, we will show that $m(A) \neq 0$ and obtain a contradiction. To this end, we claim that

$$
a_{r+q, r-1}^{(k)}= \begin{cases}(q-1)(2 r-3)+1 & \text { if } k=r, \\ 2(q-1)(r-1) & \text { if } k>r .\end{cases}
$$

When $k=r$, the number of diwalks from the vertex $r+q$ to vertex $r-1$ can be enumerated in the following three ways.

(i) If the rim vertices of $W_{q}$ are avoided.

In this case, there is only one such diwalk, namely $r+q \rightarrow r \rightarrow 1 \rightarrow 2 \rightarrow \cdots \rightarrow r-2 \rightarrow$ $r-1$.

(ii) Move along some rim vertices of $W_{q}$ with the hub vertex $r$ of $F_{r}$ being included in the diwalk.

For each rim vertex of $W_{q}$, since the hub vertex $r$ of $F_{r}$ is included in the diwalk, the possible number of rim vertices of $F_{r}$ covered in the diwalk is at most $r-2$. From the multiplication principle, since $W_{q}$ has $q-1$ rim vertices, the number of diwalks from vertex $r+q$ to vertex $r-1$ in case (ii) is $(q-1)(r-2)$.

(iii) Move along some rim vertices of $W_{q}$ with the hub vertex $r$ of $F_{r}$ being excluded in the diwalk.

In this case, since the hub vertex $r$ of $F_{r}$ is excluded in the diwalk, the possible number of rim vertices of $F_{r}$ contained in the diwalk is $r-1$. This implies that the number of diwalks from vertex $r+q$ to vertex $r-1$ in case (iii) is $(q-1)(r-1)$.

Thus, for the case $k=r, a_{r+q, r-1}^{(k)}=1+(q-1)(r-2)+(q-1)(r-1)=(q-1)(2 r-$ 3) +1 .

Likewise, the similar enumeration method for the case when $k=r$ can be used to show that $a_{r+q, r-1}^{(k)}=0+(q-1)(r-1)+(q-1)(r-1)=2(q-1)(r-1)$ when $k>r$.

By using the results in the claim, we have

$$
\begin{aligned}
a_{r+q, r-1}^{(r+q-1)}-a_{r+q, r-1}^{(r)} & =2(q-1)(r-1)-\{(q-1)(2 r-3)+1\} \\
& =q-2 \neq 0 .
\end{aligned}
$$

This implies that $m(A)=A^{r+q-1}-A^{r} \neq 0$, a contradiction. Therefore $\psi(x)$ is equal to the minimum polynomial of $W_{q} \otimes F_{r}$, and $W_{q} \otimes F_{r}$ is annihilatingly unique and the proof of Part 2 is complete. 


\section{References}

[1] D. M. Cvetković, M. Doob, and H. Sachs, Spectra of Graphs. Theory and Application, Pure and Applied Mathematics, vol. 87, Academic Press, New York, 1980.

[2] F. Harary, Graph Theory, Addison-Wesley, Massachusetts, 1969.

[3] K. S. Lam, On digraphs with unique annihilating polynomial, Ph.D. thesis, University of Malaya, Kuala Lumpur, 1990.

[4] C. K. Lim and K. S. Lam, The characteristic polynomial of ladder digraph and an annihilating uniqueness theorem, Discrete Math. 151 (1996), no. 1-3, 161-167.

[5] M. Marcus and H. Minc, A Survey of Matrix Theory and Matrix Inequalities, Allyn and Bacon, Massachusetts, 1964.

[6] E. D. Nering, Linear Algebra and Matrix Theory, John Wiley \& Sons, New York, 1963.

C. S. Gan: Faculty of Engineering and Technology, Multimedia University, 75450 Malacca, Malaysia E-mail address: csgan@mmu.edu.my 


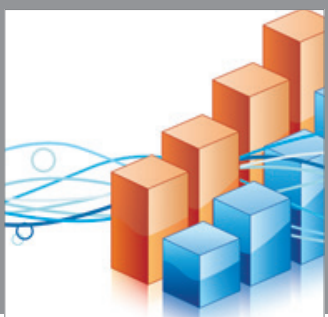

Advances in

Operations Research

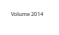

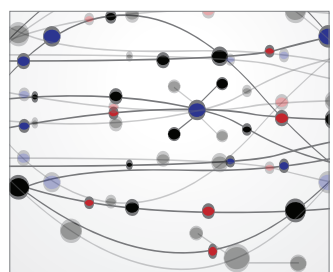

\section{The Scientific} World Journal
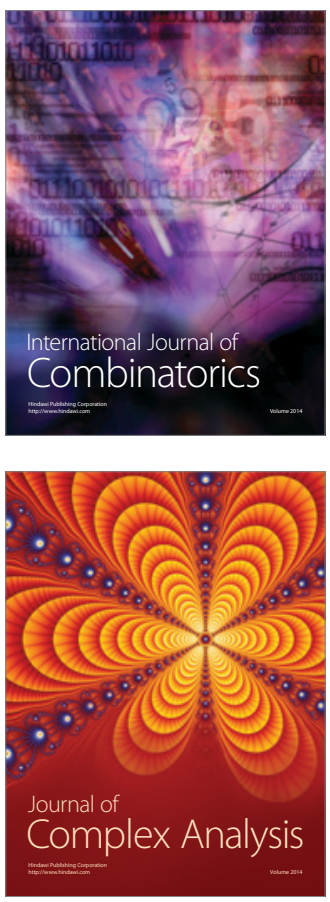

International Journal of

Mathematics and

Mathematical

Sciences
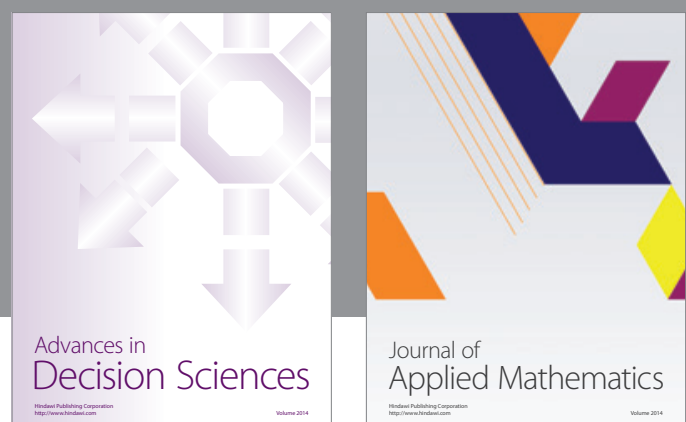

Journal of

Applied Mathematics
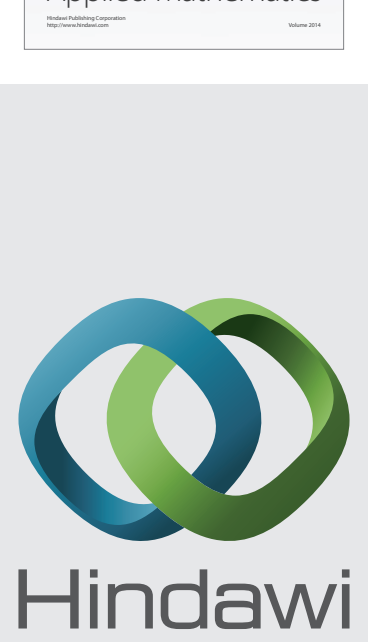

Submit your manuscripts at http://www.hindawi.com
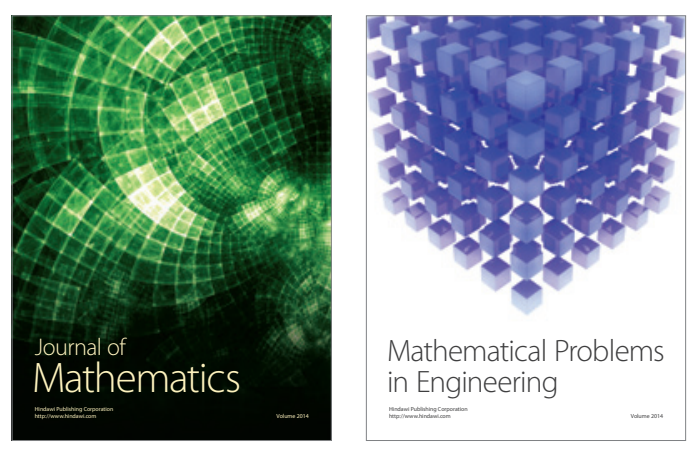

Mathematical Problems in Engineering
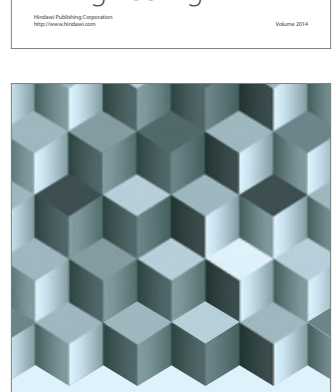

Journal of

Function Spaces
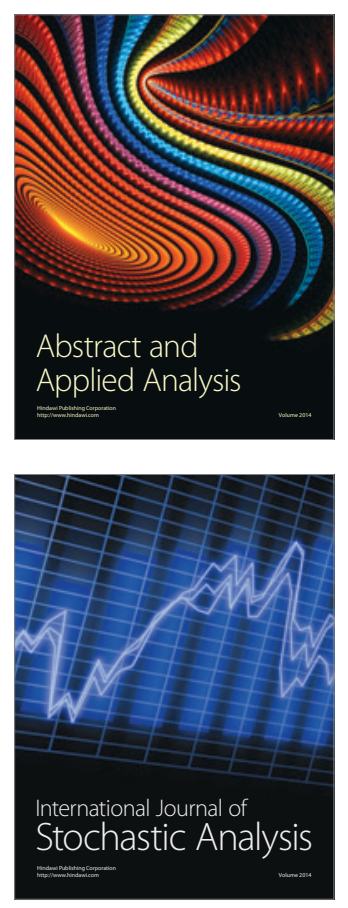

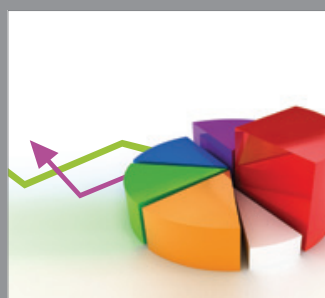

ournal of

Probability and Statistics

Promensencen
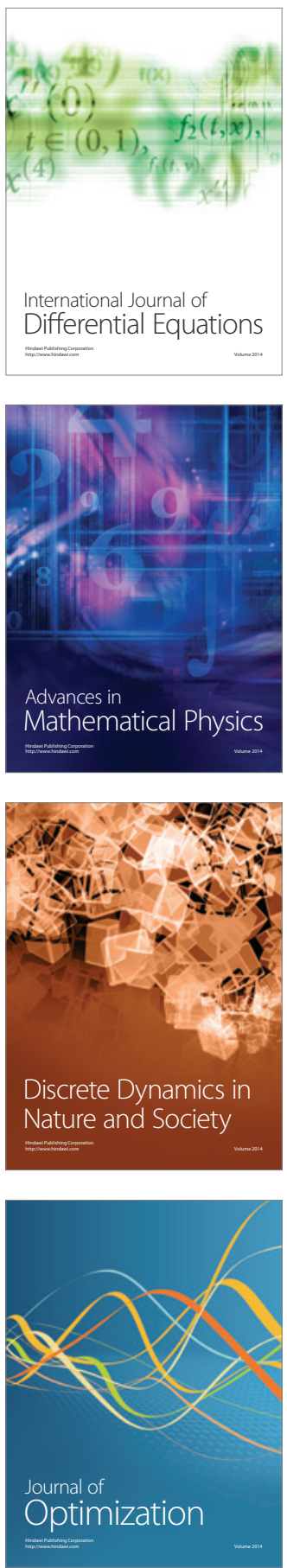